\title{
A report on the experience of using ultrasound by emergency medical technicians in dealing with trauma patients in pre-hospital setting: a pilot study
}

\author{
Pir-Hossein Kolivand ${ }^{1,2}$, Peyman Saberian ${ }^{1,3^{*}}$, Mostafa Sadeghi ${ }^{4}$, Maryam Modabber ${ }^{5}$, Parisa Hasani- \\ Sharamin ${ }^{5}$ \\ 'Prehospital and Hospital Emergency Research Center, Tehran University of Medical Sciences, Tehran, Iran \\ ${ }^{2}$ Shefa Neuroscience Research Center, Khatam-al-Anbya Hospital, Tehran, Iran \\ ${ }^{3}$ Anesthesiology Department, Imam Khomeini Hospital Complex, Tehran University of Medical Sciences, Tehran, Iran \\ ${ }^{4}$ Anesthesiology Department, Shariati Hospital, Tehran University of Medical Sciences, Tehran, Iran \\ ${ }^{5}$ Tehran Emergency Medical Service Center, Tehran, Iran
}

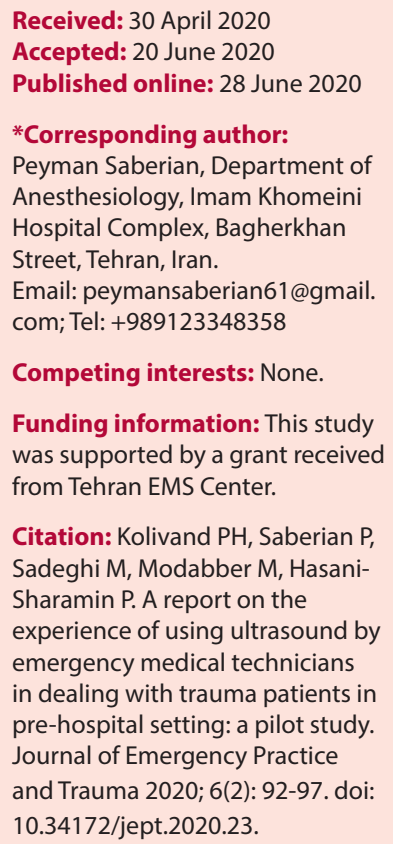

Citation: Kolivand PH, Saberian $\mathrm{P}$ Sadeghi M, Modabber M, HasaniSharamin P. A report on the experience of using ultrasound by emergency medical technicians in dealing with trauma patients in pre-hospital setting: a pilot study. Journal of Emergency Practice and Trauma 2020; 6(2): 92-97. doi: 10.34172/jept.2020.23.

\begin{abstract}
Objective: The current study was performed to provide real-time bedside ultrasonography for emergency medical technicians (EMTs) and assess the advantages and disadvantages of its application in dealing with trauma patients in pre-hospital setting from their viewpoints.

Methods: This semi-experimental study was conducted in Tehran, Iran. Twenty EMTs were selected purposefully and underwent a training program. Thereafter, they were asked to perform extended focused assessment with sonography in trauma (eFAST) using a handheld ultrasound device on trauma patients, and also filled a questionnaire prepared (in four components including C1: coherence, C2: cognitive participation, C3: collective action, and C4: reflexive monitoring) based on the normalization process theory (NPT). Results: All 20 participants were men and their average age was 37.8 years ( $S D=4.7)$. For $\mathrm{C} 1$, the median total score was 10.5 out of a score of 4-20; For $\mathrm{C} 2$, the median score was 6 out of 3-15; For C3, the median total score was 18 out of a score of 6-30; and for C4, the median total score was 11 out of a score of 5-25.

Conclusion: Overall, it seems that EMTs welcomed using ultrasonography in dealing with trauma patients in pre-hospital setting. Although they thought that it might somewhat lead to an increase in their workload; but they believed that sufficient training was not provided for them yet. The EMTs were uncertain about the viewpoints of the patients and did not know how it could affect patients' outcome.

Keywords: Emergency medical services, Focused assessment with sonography for trauma, Multiple trauma, Ultrasonography
\end{abstract}

\section{Introduction}

Downsizing of the ultrasound devices has made it possible to make more use of it; Not only the patients no longer need to be moved to a separate room for performing ultrasonography, but also the ultrasound machine is easily brought to the patient's bedside. This has allowed for defining more applications of ultrasound in dealing with emergency patients (1). One of its most common roles has been defined in primary surveys of trauma patients as "extended focused assessment with sonography in trauma (eFAST)", that is performed routinely in the emergency department (ED) (2-4). Recently, Pre-hospital Trauma Life Support (PHTLS) guidelines have attempted to increase the role of pre-hospital emergency care in trauma patients, whether in terms of therapeutic interventions or diagnostic measures (5). So, the possibility of using ultrasound for performing eFAST in the pre-hospital phase has been raised and its effectiveness, advantages, and disadvantages have been challenged $(3,6)$. It seems that pre-hospital ultrasound studies started around the year 2000 and primarily concentrated on using FAST on the aeromedical field. This has led to contradictory results in terms of feasibility, usefulness and etc $(7,8)$. Later, more studies were designed concerning the potential benefits of its use in ground missions, on different patients, and of course, with different applications. Overall, it is believed that pre-hospital ultrasound alters the diagnosis and management of patients, but there is a paucity of evidence regarding patients' outcomes (5). In Iran, portable ultrasound devices have not yet been deployed 
in ambulances; But the idea has caught the attention of administrators. Therefore, the current study was performed to provide real-time bedside ultrasonography for emergency medical technicians (EMTs) and assess the advantages and disadvantages of its application in dealing with trauma patients in pre-hospital setting from their viewpoints.

\section{Methods}

This semi-experimental study was performed from June 16, 2019 to August 25, 2019 in Tehran, Iran.

Twenty EMTs were selected purposefully from those who had a bachelor's degree and at least a 2-year work experience. They underwent a training program consisting of 4 hours of theory and 4 hours of practical workshops. Then, all the EMTs performed eFAST once on a healthy person under the supervision of an emergency medicine specialist to get the required certification for performing eFAST at the scene. The selected EMTs were asked to perform eFAST using a handheld ultrasound device on trauma patients and record the images. The saved images were reviewed by an emergency medicine specialist in terms of quality and accuracy. In addition, the results of the review of the images (feedback) were communicated with the EMTs and they filled a questionnaire prepared based on the normalization process theory (NPT) in four components including coherence, cognitive participation, collective action, and reflexive monitoring. The NPT model is a conceptual tool proposed to help in understanding the factors that affect implementation processes in clinical trials and other evaluations of complex interventions. It focuses on the ways that the implementation of complex interventions is shaped by problems of workability and integration $(9,10)$. In the end, the EMTs were free to mention their opinion regarding the advantages and disadvantages of using sonography in the pre-hospital setting.

The descriptive analysis was conducted and frequency (with percentage), mean (with standard deviation, SD), median and quartile were reported as appropriate. For comparing the strengths and weaknesses of ultrasound for different domains, we calculated standard score (0-100) based on the number of questions for each domain. The standard score was the raw score divided by the number of questions multiplied by 100 .

\section{Results}

All 20 participants were males and the average age was 37.8 years $(\mathrm{SD}=4.7)$. In terms of work experience, $2,5,7$ and 6 participants had a work experience of 3-5 years, 6-10 years, 11-15 years and more than 15 years, respectively. The mean score of feeling familiar with pre-hospital ultrasound was $6.42(\mathrm{SD}=1.50)$. Also, the mean score of feeling that pre-hospital ultrasound will be a routine part of their work now and in the future was $3.11(\mathrm{SD}=$
$2.21)$ and $5.47(\mathrm{SD}=2.14)$, respectively. Table 1 shows the frequency distribution of each of the questions related to awareness, attitude, as well as the practicality nature of pre-hospital ultrasound.

For C1, the majority of individuals (42.1\%) received a score of 9 out of a score of 4-20, and the median total score was 10.5 . For C2, the majority of individuals (38.9\%) received a score of 3 out of 3-15, and the median score was 6. For C3, the majority of individuals (33.3\%) received a score of 18 out of a score of 6-30, and the median total score was 18 . For C4, the majority of individuals (31.6\%) received a score of 7 out of a score of 5-25, and the median total score was 11 (Figure 1).

Weaknesses and strengths of ultrasound regarding workload, diagnosis, management, training, practicality, patients, and early notification were studied. All areas had a combination of positive and negative questions, using a 5-point Likert scale (0-4), and a higher total score indicated more strength. The mean score for the workload with 11 questions was $17.15(\mathrm{SD}=6.85)$ and the standardized mean score in terms of the number of questions for the workload was $31.18(\mathrm{SD}=12.46)$. The frequency distribution of raw and standardized scores is presented in Table 2 and Figure 2. The greatest benefit of ultrasound-based on the standardized score was for patients, management, and diagnosis.

Regarding the workload, the most important strength (highest score) and weakness (lowest score) were respectively, "Short time to perform the ultrasound" with the mean score of 2.4 and "Increase of responsibility without increasing of incomeor support" with the mean score of 0.75 . Regarding the diagnosis, the most important strength (highest scores) was "It can be very helpful in diagnosing a very ill patient" and "It is effective in diagnosing pneumothorax in a noisy environment" with the mean score of 2.6, and the most important weakness (lowest score) was "It is very dangerous if it is used as a definitive diagnostic method/can easily be misinterpreted" with the mean score of 1.3. Concerning the management, the most important strength (highest score) and weakness (lowest scores) were respectively "its role in crises or cases far from the facilities is significant" with the mean score of 3.1 and "it does not make a difference for cases like fractures" with the mean score of 1.4. In terms of training, the most important strength (highest scores) and weakness (lowest scores) were respectively, "Ultrasound training motivates the staff", with the mean score of 3.2 and "One-day training is not enough for the ultrasound" with the mean score of 0.45 . Regarding the practicality, the most important strength (highest score) and weakness (lowest score) were respectively "portability" with the mean score of 3.1 and "too time-consuming to perform ultrasound" with the mean score of 1.4. With regard to the patients, the most important strength (highest scores) and weakness (lowest scores) were respectively "When 
Table 1. Frequency distribution of responses related to awareness, attitude as well as the practicality of pre-hospital ultrasound

\begin{tabular}{|c|c|c|c|c|c|}
\hline & $\begin{array}{l}\text { Strongly } \\
\text { agree }\end{array}$ & Agree & Neutral & Disagree & $\begin{array}{l}\text { Strongly } \\
\text { disagree }\end{array}$ \\
\hline \multicolumn{6}{|l|}{ Part C1: coherence } \\
\hline Pre-hospital ultrasound is different from my current routine. $(n=18)$ & $4(22.2)$ & $9(50.0)$ & $2(11.1)$ & $3(16.7)$ & 0 \\
\hline $\begin{array}{l}\text { All EMS staff have a common understanding of the purpose of pre-hospital } \\
\text { ultrasound. }(n=19)\end{array}$ & 0 & $2(10.5)$ & $2(10.5)$ & $10(52.6)$ & $5(26.3)$ \\
\hline Pre-hospital ultrasound affects the nature of my work. $(n=19)$ & $5(26.3)$ & $10(52.6)$ & $2(10.5)$ & $1(5.3)$ & $1(5.3)$ \\
\hline $\begin{array}{l}\text { I understand the potential value that pre-hospital ultrasound has for my work. } \\
(\mathrm{n}=19)\end{array}$ & $3(15.8)$ & $11(57.9)$ & $4(21.1)$ & 0 & $1(5.3)$ \\
\hline \multicolumn{6}{|l|}{ Part C2: cognitive participation } \\
\hline $\begin{array}{l}\text { I believe that participating in pre-hospital ultrasound is the right part of my role. } \\
(n=18)\end{array}$ & $7(38.9)$ & $5(27.8)$ & $2(11.1)$ & $3(16.7)$ & $1(5.6)$ \\
\hline I welcome the role of my colleagues in the use of pre-hospital ultrasound. $(n=18)$ & $7(38.9)$ & $5(27.8)$ & $2(11.1)$ & $3(16.7)$ & $1(5.6)$ \\
\hline I will support the pre-hospital ultrasound. $(n=18)$ & $7(38.9)$ & $5(27.8)$ & $1(5.6)$ & $4(22.2)$ & $1(5.6)$ \\
\hline \multicolumn{6}{|l|}{ Part C3: collective action } \\
\hline I can easily integrate pre-hospital ultrasound into my existing work. ( $n=17)$ & $2(11.8)$ & $9(52.9)$ & $4(23.5)$ & $2(11.8)$ & 0 \\
\hline Pre-hospital ultrasound disrupts the work process. $(n=18)$ & $1(5.6)$ & $3(16.7)$ & $3(16.7)$ & $6(33.3)$ & $5(27.8)$ \\
\hline I trust the ability of others to use pre-hospital ultrasound. $(n=18)$ & 0 & $3(16.7)$ & $11(61.1)$ & $2(11.1)$ & $2(11.1)$ \\
\hline $\begin{array}{l}\text { Adequate training has been provided to empower staff to perform the pre- } \\
\text { hospital ultrasound. }(n=17)\end{array}$ & 0 & $3(17.6)$ & $5(29.4)$ & $5(29.4)$ & $4(23.5)$ \\
\hline Sufficient resources are available for the pre-hospital ultrasound. $(n=17)$ & 0 & $4(23.5)$ & $3(17.6)$ & $5(29.4)$ & $5(29.4)$ \\
\hline The manager sufficiently supports pre-hospital ultrasound. $(n=17)$ & $1(5.9)$ & $4(23.5)$ & $9(52.9)$ & $1(5.9)$ & $2(11.8)$ \\
\hline \multicolumn{6}{|l|}{ Part C4: reflexive monitoring } \\
\hline I am aware of the positive effects of using pre-hospital ultrasound. $(n=18)$ & $4(22.2)$ & $8(44.4)$ & $2(11.1)$ & $1(5.6)$ & $3(16.7)$ \\
\hline The staff agrees that pre-hospital ultrasound is valuable. $(n=19)$ & $2(10.5)$ & $7(36.8)$ & $7(36.8)$ & $2(10.5)$ & $1(5.3)$ \\
\hline $\begin{array}{l}\text { The effects that pre-hospital ultrasound has on my work are important to me. } \\
(n=19)\end{array}$ & $8(42.1)$ & $7(36.8)$ & $1(5.3)$ & $2(10.5)$ & $1(5.3)$ \\
\hline $\begin{array}{l}\text { Staff feedback on pre-hospital ultrasound can be used to improve it in the future. } \\
\text { ( } n=19 \text { people) }\end{array}$ & $6(31.6)$ & $7(36.8)$ & $5(26.3)$ & 0 & $1(5.3)$ \\
\hline I can improve my way of working with pre-hospital ultrasound. ( $n=18)$ & $7(38.9)$ & $9(50.0)$ & $1(5.6)$ & 0 & $1(5.6)$ \\
\hline
\end{tabular}

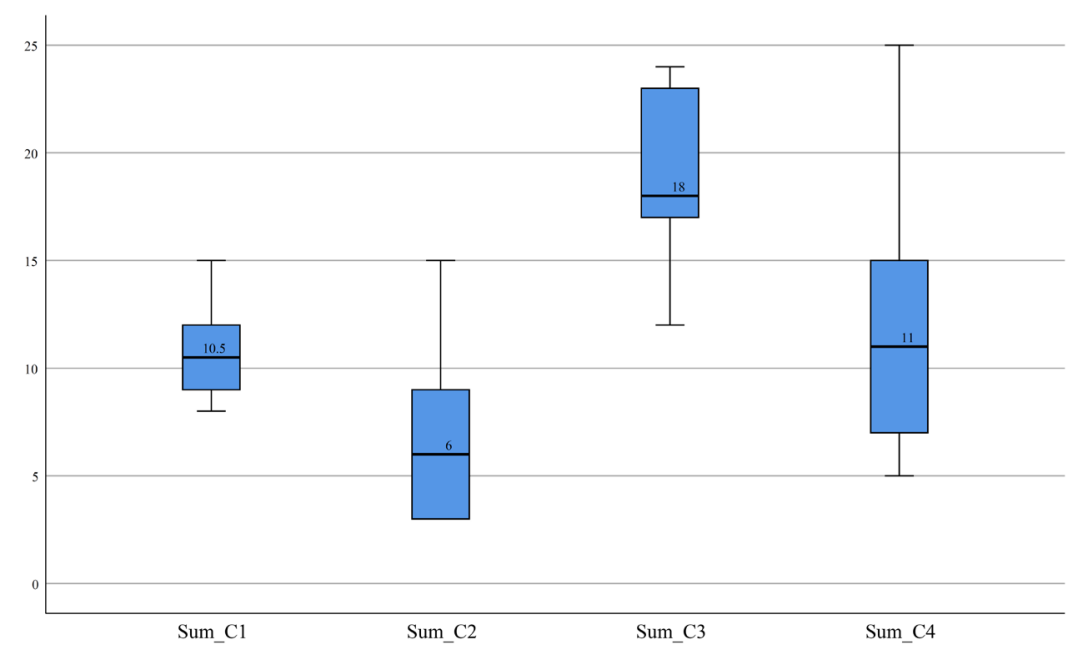

Figure 1. The median score of four components of study questionnaire (C1: coherence, C2: cognitive participation, C3: collective action, C4: reflexive monitoring). 
Table 2. The raw and standardized score of pros and cons of pre-hospital ultrasound for different areas

\begin{tabular}{|c|c|c|c|c|c|c|c|c|c|c|}
\hline & \multirow{2}{*}{$\begin{array}{l}\text { Number of } \\
\text { Questions }\end{array}$} & \multirow{2}{*}{$\begin{array}{l}\text { Raw score } \\
\text { range }\end{array}$} & \multicolumn{4}{|l|}{ Raw score } & \multicolumn{4}{|c|}{ Standardized score $(0-100)$} \\
\hline & & & Mean (SD) & Q1 & Q2= Median & Q3 & Mean (SD) & Q1 & Q2= Median & Q3 \\
\hline Workload & 11 & $0-44$ & $17.15(6.85)$ & 14.00 & 20.00 & 21.00 & $31.18(12.46)$ & 25.45 & 36.36 & 38.18 \\
\hline Diagnosis & 13 & $0-52$ & $26.80(9.96)$ & 24.00 & 28.50 & 32.00 & $41.23(15.32)$ & 36.92 & 43.85 & 49.23 \\
\hline Management & 9 & $0-36$ & $20.10(6.65)$ & 14.25 & 22.00 & 23.75 & 44.67 (14.77) & 31.67 & 48.89 & 52.78 \\
\hline Training & 9 & $0-36$ & $14.00(3.55)$ & 12.25 & 15.00 & 16.00 & $31.11(7.90)$ & 27.22 & 33.33 & 35.56 \\
\hline Practicality & 11 & $0-44$ & $19.00(6.30)$ & 13.50 & 18.50 & 25.00 & 34.55 (11.45) & 24.55 & 33.64 & 45.45 \\
\hline Patients & 6 & $0-24$ & $13.47(4.76)$ & 11.00 & 15.00 & 16.50 & $44.90(15.86)$ & 36.67 & 50.00 & 55.00 \\
\hline Early notification & 6 & $0-24$ & $11.06(4.79)$ & 7.50 & 12.00 & 14.00 & $36.86(15.96)$ & 25.00 & 40.00 & 46.67 \\
\hline
\end{tabular}

SD: Standard deviation; Q: Quartile.

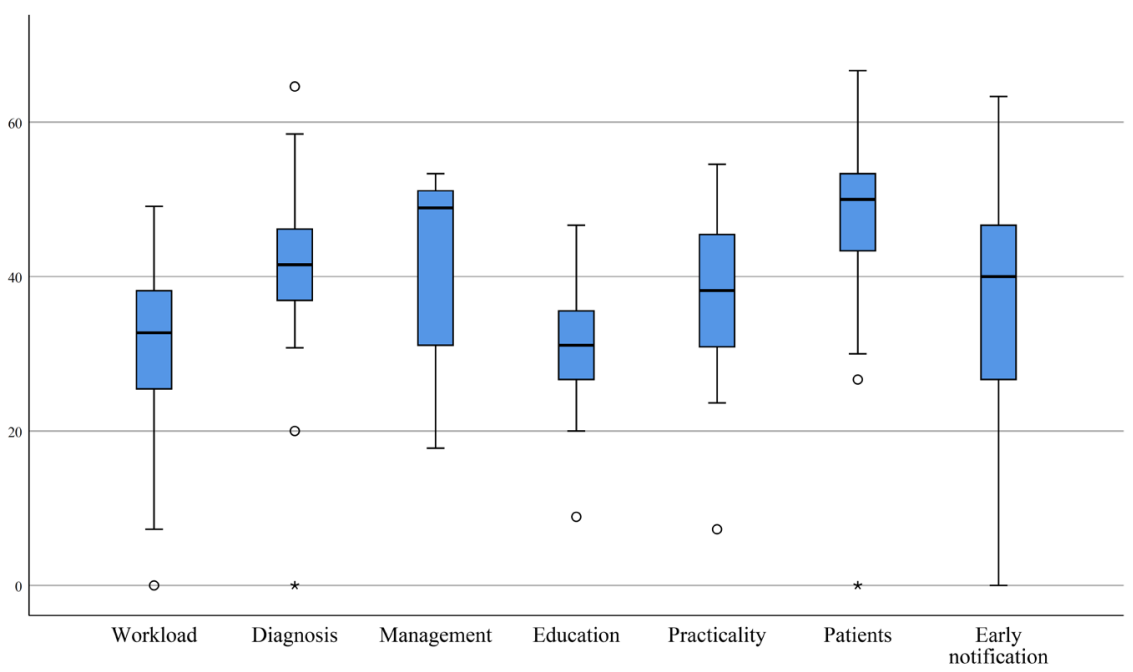

Figure 2. Frequency distribution of pros and cons score for different areas of using ultrasound in pre-hospital setting.

I explain to patients, they realize that ultrasound is a standard of care" with the mean score of 2.8 and "patients may not want to have an ultrasound" with the mean score of 1.7. Concerning the notification, the most important strength (highest score) and weakness (lowest score) were respectively "Quick notification to the hospital ensures that appropriate and definite facilities and resources are provided to the patient faster" with the mean score of 2.6 and "There is not enough confidence in pre-hospital notification" with the mean score of 1.1.

\section{Discussion}

The present study, after a short experience of using ultrasound in the pre-hospital phase, evaluated its pros and cons in various aspects. Overall, it seems that EMTs welcomed its use, even though they thought that it might somewhat lead to an increase in their workload. Moreover, they believed that sufficient training was not provided for them. The interviewees were uncertain about the viewpoints of the patients as well as the how it could affect the patients' outcome. But they considered it practical, and were aware of the support and desire of the management system to use ultrasound in the ambulances. This may be due to the lack of a classification of trauma centers in some areas, as well as the limited number of appropriate hospitals in case the technician detects positive FAST. Also, in some cases, because of the short time of transfer (especially in air medical transport), there is not enough time to use the ultrasound. Therefore, the pre-hospital ultrasound is recommended in cases of far distance to the hospital and there is also the possibility of triage to select the appropriate hospital.

In a study conducted by Walcher et al, it was reported that the accuracy of FAST performed in the pre-hospital phase was about $99 \%$ and the results ultimately led to a change in the management of about $30 \%$ of patients. However, it took only about 2-3 minutes to complete the FAST (11). It should be mentioned that in Walcher and colleagues' study, physicians or paramedics who underwent proper training courses performed the FAST. But, in Iran, there is no physician or paramedic in EMS ambulances, and all EMTs have the same degree with various work experiences. It 
seems that the managers should consider this point before the establishment of the ultrasound devices in ambulances or at least before running a project and provide adequate training courses.

One of the points raised by the participants in the present study was that how pre-hospital ultrasonography could affect patients' outcome. There are several case reports that indicate the usefulness of pre-hospital ultrasonography in detecting tamponade in patients with penetrating chest trauma which result in either pericardiocentesis or improved triage process of the patients $(12,13)$. Its usefulness was introduced not only in terms of performing advanced procedures but also in avoiding unnecessary procedures (14). Although pre-hospital ultrasound may not change the pre-hospital management of the most common conditions, it could be life-saving in some instances. Therefore, it is better to define and determine its uses prior to its general application in the system. By reviewing current literature, we found a pilot study conducted by Chin et al. in which 20 EMT-paramedics with no prior ultrasonography training underwent a brief course (a 2-hour training session) and thereafter were tested on image acquisition skills as well as image interpretation for several life-threatening conditions such as pneumothorax, pericardial effusion, and cardiac activity. They reported that the enrolled EMT-paramedics were able to perform the Prehospital Assessment with UltraSound for Emergencies (PAUSE) protocol (15). There are some other published papers, which reported that EMS physicians and paramedics without prior ultrasonography training can be trained effectively after a short course (16-19).

Overall, it seems that ultrasound in Iran, like many developed countries, is going to be widely used in the prehospital emergency system (20). But the structure of this organization in our country is very different from that of the rest of the world, and naturally, different planning needs to be designed in order to make the best use of this tool in the pre-hospital setting.

\section{Limitations}

In the current study, we did not assess the learning curve of the participated EMTs. Also, the results of the performed eFAST did not alter the patients' outcome, so we cannot discuss it in this regard.

\section{Conclusion}

The EMTs participated in the current study, generally, welcomed using ultrasonography in pre-hospital setting for dealing with trauma patients; but they thought that sufficient training is required before its general establishment in ambulances. They also had concern about its effect on patients' management and outcome.

\section{Authors' contributions}

The conception and design of the work by PHK, PS and
PHS; Data acquisition by FD, SB and EA; Analysis and interpretation of data by PS, PHS and SB; Drafting the work by FD, SB and EA; Revising it critically for important intellectual content by PHK, PS, and PHS; All the authors approved the final version to be published; and agree to be accountable for all aspects of the work in ensuring that questions related to the accuracy or integrity of any part of the work.

\section{Ethical issues}

The study proposal was approved by the ethics committee of Tehran University of Medical Sciences (code: IR.TUMS. VCR.REC.1397.1065) and the investigators fully adhered to the Declaration of Helsinki Principles throughout the study.

\section{Acknowledgements}

We would like to express our obligations to Pre-hospital and Hospital Emergency Research Center affiliated to Tehran University of Medical Sciences.

\section{References}

1. Abdolrazaghnejad A, Banaie M, Safdari M. Ultrasonography in emergency department; a diagnostic tool for better examination and decision-making. Adv J Emerg Med 2018; 2(1): e7. doi: 10.22114/AJEM.v0i0.40.

2. Heydari F, Ashrafi A, Kolahdouzan M. Diagnostic accuracy of focused assessment with sonography for blunt abdominal trauma in pediatric patients performed by emergency medicine residents versus radiology residents. Adv J Emerg Med 2018; 2(3): e31. doi: 10.22114/AJEM.v0i0.89.

3. Samuel AE, Chakrapani A, Moideen F. Accuracy of extended focused assessment with sonography in trauma (e-FAST) performed by emergency medicine residents in a level one tertiary center of India. Adv J Emerg Med 2018; 2(2): e15. doi: 10.22114/ajem.v0i0.69.

4. Bagheri-Hariri S, Bahreini M, Farshidmehr P, Barazandeh S, Babaniamansour S, Aliniagerdroudbari E, et al. The effect of extended-focused assessment with sonography in trauma results on clinical judgment accuracy of the physicians managing patients with blunt thoracoabdominal trauma. Arch Trauma Res 2019;8(4):207-13. doi: 10.4103/ atr.atr_57_19.

5. Roantree RAG, Furtado CS, Lambert MJ. EMS, Ultrasound Use. In: StatPearls. Treasure Island, FL: StatPearls Publishing; 2020.

6. El Zahran T, El Sayed MJ. Prehospital ultrasound in trauma: a review of current and potential future clinical applications. J Emerg Trauma Shock 2018; 11(1): 4-9. doi: 10.4103/jets. jets_117_17.

7. Price DD, Wilson SR, Murphy TG. Trauma ultrasound feasibility during helicopter transport. Air Med J 2000; 19(4): 144-6. doi: 10.1016/s1067-991x(00)90008-7.

8. Melanson SW, McCarthy J, Stromski CJ, Kostenbader J, Heller M. Aeromedical trauma sonography by flight crews with a miniature ultrasound unit. Prehosp Emerg Care 2001; 5(4): 399-402. doi: 10.1080/10903120190939607.

9. Murray E, Treweek S, Pope C, MacFarlane A, Ballini L, Dowrick C, et al. Normalisation process theory: a framework for developing, evaluating and implementing 
complex interventions. BMC Med 2010; 8: 63. doi: 10.1186/1741-7015-8-63.

10. May C, Rapley T, Mair FS, Treweek S, Murray E, Ballini L, et al. Normalization Process Theory On-line Users' Manual, Toolkit and NoMAD instrument. 2015. Available from: http://www.normalizationprocess.org/resources/. Accessed 28 May 2019.

11. Walcher F, Weinlich M, Conrad G, Schweigkofler U, Breitkreutz R, Kirschning T, et al. Prehospital ultrasound imaging improves management of abdominal trauma. $\mathrm{Br} \mathrm{J}$ Surg 2006; 93(2): 238-42. doi: 10.1002/bjs.5213.

12. Byhahn C, Bingold TM, Zwissler B, Maier M, Walcher F. Prehospital ultrasound detects pericardial tamponade in a pregnant victim of stabbing assault. Resuscitation 2008; 76(1): 146-8. doi: 10.1016/j.resuscitation.2007.07.020.

13. Heegaard W, Hildebrandt D, Reardon R, Plummer D, Clinton J, Ho J. Prehospital ultrasound diagnosis of traumatic pericardial effusion. Acad Emerg Med 2009; 16(4): 364. doi: 10.1111/j.1553-2712.2009.00379.x.

14. Roberts J, McManus J, Harrison B. Use of ultrasonography to avoid an unnecessary procedure in the prehospital combat environment: a case report. Prehosp Emerg Care 2006; 10(4): 502-6. doi: 10.1080/10903120600887023.

15. Chin EJ, Chan CH, Mortazavi R, Anderson CL, Kahn CA, Summers S, et al. A pilot study examining the viability of a
Prehospital Assessment with UltraSound for Emergencies (PAUSE) protocol. J Emerg Med 2013; 44(1): 142-9. doi: 10.1016/j.jemermed.2012.02.032.

16. Lyon M, Walton P, Bhalla V, Shiver SA. Ultrasound detection of the sliding lung sign by prehospital critical care providers. Am J Emerg Med 2012; 30(3): 485-8. doi: 10.1016/j.ajem.2011.01.009.

17. Krogh CL, Steinmetz J, Rudolph SS, Hesselfeldt R, Lippert FK, Berlac PA, et al. Effect of ultrasound training of physicians working in the prehospital setting. Scand J Trauma Resusc Emerg Med 2016; 24: 99. doi: 10.1186/ s13049-016-0289-1.

18. Gracias VH, Frankel HL, Gupta R, Malcynski J, Gandhi R, Collazzo L, et al. Defining the learning curve for the Focused Abdominal Sonogram for Trauma (FAST) examination: implications for credentialing. Am Surg 2001; 67(4): 364-8.

19. Bobbia X, Hansel N, Muller L, Claret PG, Moreau A, Genre Grandpierre R, et al. Availability and practice of bedside ultrasonography in emergency rooms and prehospital setting: a French survey. Ann Fr Anesth Reanim 2014; 33(3): e29-33. doi: 10.1016/j.annfar.2013.12.010.

20. Ketelaars R, Reijnders G, van Geffen GJ, Scheffer GJ, Hoogerwerf N. ABCDE of prehospital ultrasonography: a narrative review. Crit Ultrasound J 2018; 10(1): 17. doi: 10.1186/s13089-018-0099-y. 